\title{
Age-related BMAL1 change affects mouse bone marrow stromal cell proliferation and osteo-differentiation potential
}

\author{
Yijia Chen ${ }^{1,2}$, Xiaomei Xu', Zhen Tan ${ }^{2,4}$, Cui Ye ${ }^{1,2}$, Qing Zhao ${ }^{1,2}$, Yangxi Chen ${ }^{1,2}$
}

1Orthodontic Centre, West China College of Stomatology, Sichuan University, Chengdu, China

2State Key Laboratory of Oral Diseases, Sichuan University, Chengdu, China

3Department of Orthodontics, Stomatological Hospital of Luzhou Medical College,

Luzhou, China

${ }^{4}$ Oral Implant Centre, West China College of Stomatology, Sichuan University, Chengdu, China

Submitted: 19 October 2010

Accepted: 24 February 2011

Arch Med Sci 2012; 8, 1: 30-38

DOI: $10.5114 /$ aoms.2012.27277

Copyright $\odot 2012$ Termedia \& Banach

\begin{abstract}
Introduction: Aging people's bone regeneration potential is always impaired. Bone marrow stromal cells (MSCs) contain progenitors of osteoblasts. Donor age may affect MSCs' proliferation and differentiation potential, but the genomic base is still unknown. Due to recent research's indication that a core circadian component, brain and muscle ARNT-like 1 protein (BMAL1), has a role in premature aging, we investigated the normal aging mechanism in mice with their MSCs and Bmal1 gene/protein level.

Material and methods: 1, 6 and 16 month old C57BL/ 6 mice were used and the bone marrow stromal cells were gained and cultured at early passage. Bmal1 gene and protein level were detected in these cells. Marrow stromal cells were also induced to differentiate to osteoblasts or adipocytes. Three groups of mice MSCs were compared on proliferation by flow cytometry, on cell senescence by SA- $\beta$-gal expression and after osteo-induction on osteogenic potential by the expression of osterix (Osx), alkaline phosphatase (ALP) and osteocalcin (OCN). Results: Bmal1 gene and protein level as well as S-phase fraction of the cell cycle decreased in MSCS along with the aging process. At the same time, SA- $\beta$-gal+ levels increased, especially in the aged mice MSCs. When induced to be osteogenic, Osx gene expression and ALP activity declined in the mid-age and aged mice MSCs, while OCN protein secretion deteriorated in the aged mice MSCs.

Conclusions: These findings demonstrate that mouse MSCs changed with their proliferation and osteo-differentiation abilities at different aging stages, and that Bmal1 is related to the normal aging process in MSCs.
\end{abstract}

Key words: brain and muscle ARNT-like 1 protein, bone marrow stromal cells, aging, differentiation, proliferation.

\section{Introduction}

In the elderly, consequences of teeth loss for the maxilla and mandible are well known [1]. They are manifested by loss of jaw bone, especially bone that forms the socket of the teeth on the jaw, which is termed "alveolar bone", the same as other bones in the human body. The poor bone regeneration potential will prolong or even ruin the result of orthodontic

\author{
Corresponding author: \\ Qing Zhao \\ No. 14 , Section 3 \\ Renmin Nan Road \\ Chengdu, Sichuan \\ 610041, China \\ Phone: +86 02885502207 \\ E-mail: fanfan.qing@ \\ gmail.com
}


treatment for adult and elderly people. Losing bone can cause an aged morphological change on the face and impair possible implant or denture placement. The aged bone loss is due to the diminished activity of bone forming cells. Osteoblasts are derived from progenitors residing in the bone stroma. These cells are normally called bone marrow stromal cells (MSCs) [2]. When aging, while MSCs still have self-renewal ability and multi-differentiation ability, these abilities, but the mechanism of the aging change is still being explored.

Recently, studies have focused on BMAL1, a core component of circadian rhythm, concerning its role in the aging process of mammals [3, 4]. Bmal-/mice show early aging symptoms compared with their wild-type littermates. These symptoms include decreased life span, arthropathy, osteoporosis, etc., and the same defects do not occur in other circadian gene knock-out animals. Researchers place BMAL1 as another osteo-differentiation-related gene changing with age, highlighting its non-circadian functions. While formerly found in the suprachiasmatic nucleus (SCN) and controlling daily rhythm, circadian genes are also involved in control of homeostasis in peripheral tissues and organs. The peripheral clocks, such as in the liver, skeletal muscle, etc, while synchronized with the central circadian pacemaker, can play a tissue-specific role independently from the SCN [5]. Circadian genes are expressed in many kinds of cells in mammalian tissues, including the bone marrow stromal cells [6].

While early-aging symptoms of Bmal1-/- mice aroused great interest, the normal aging changes of Bmal1 expression in MSCs or other cells related to their vitality are still unknown. Bmal1's role in aging osteogenesis change of MSCs is also lacking any evidence.

The purpose of the present study was to investigate the age-related effects in mouse MSCs, namely proliferation and osteo-differentiation potential as well as the relationship between these changes and Bmal1 expression.

\section{Material and methods}

\section{Animals and cell culture}

Young adult (1 month old), middle-aged (6 months old) and aged (16 months old) male C57BL/ 6 mice were obtained from the Animal Centre of Sichuan University and the operations in the study have been approved by the University's Bioethics Committee. Animals were housed with free access to water and were maintained at a constant temperature on a 12-h light-dark cycle.

The cell isolation method was reported previously [7]. Briefly, both femora and tibias were removed and soft tissues were detached. The metaphyses from both ends of the bone were resected and the marrow cavities were flushed with sterile medium using a \#25 gauge needle. After washing with phosphate buffered saline (PBS) by centrifuge, the culture was established in T25 flasks in fresh alpha-MEM (Gibco) with 10\% fetal bovine serum (Hyclone), $100 \mathrm{U} / \mathrm{ml}$ penicillin, and $100 \mathrm{mg} / \mathrm{ml}$ streptomycin (as basic medium). Cells were maintained at $37^{\circ} \mathrm{C}$ in a humidified atmosphere containing $5 \%$ $\mathrm{CO}_{2}$. After $48 \mathrm{~h}$, the non-adherent cells were removed by gentle rinsing with sterile PBS which was pre-warmed to $37^{\circ} \mathrm{C}$, and the cells maintained in basic medium with 2 3 times per week medium change until confluent. When confluent, the MSCs were passaged by $0.25 \%$ trypsin and $0.01 \%$ ethylenediaminetetraacetic acid (EDTA). Cells of passage 3 or 4 were used in the following experiments.

\section{Marrow stromal cells identification, osteo- and adipo-differentiation procedure}

Marrow stromal cells were identified as CD29(+), Stro-1(+), CD45(-) and CD34(-) by cell immunohistochemistry as other studies suggested [8]. Confluent cultures of MSCs were induced to undergo osteogenesis by basic medium supplemented with $10 \mathrm{nM}$ dexamethasone, $10 \mathrm{mM} \beta$-glycerophosphate, and $50 \mu \mathrm{g} / \mathrm{ml} \mathrm{L}$-ascorbate (all from Sigma, as OS medium). After 21 days in OS medium, the osteo cultures were rinsed in $0.9 \% \mathrm{NaCl}$, fixed in $70 \%$ ethanol, and stained with $1 \%$ Alizarin Red for detection of calcium phosphate mineralization. The cultures were also processed with routine von Kossa staining.

Marrow stromal cells were induced to undergo adipogenesis by replacing the basic medium with adipocyte induction medium (as AD medium) composed of $\alpha$-MEM with $10 \%$ fetal bovine serum (FBS), supplemented with $100 \mathrm{nM}$ dexamethasone, $0.5 \mathrm{mM}$ isobutylmethylxanthine (IBMX), $10 \mu \mathrm{g} / \mathrm{ml}$ insulin, $0.2 \mathrm{mM}$ indomethacin, $100 \mathrm{U} / \mathrm{ml}$ penicillin, and $100 \mathrm{mg} / \mathrm{ml}$ streptomycin. After 3 days of culture with $A D$ medium, the medium was replaced with $A D$ medium without IBMX and indomethacin up to 10 days for maintenance. After that, fixed cells were stained with $0.5 \%$ oil red $\mathrm{O}$ for $1 \mathrm{~h}$ for observation of adipogenesis.

\section{Flow cytometry assay}

When passage 2 cells reached $80 \%$ confluence, they were passaged $1: 2$ to a T25 flask. After 3 days, the cells were detached with trypsin and EDTA, twice centrifuged at $500 \mathrm{~g}$ for $5 \mathrm{~min}$ containing PBS wash and then fixed with $70 \%$ alcohol overnight. Then the S-phase fraction of total cells (SPF) in each sample was analysed and calculated through flow cytometry (Beckman, US) and according to the formula: $\operatorname{SPF}(\%)=\mathrm{S} /\left(\mathrm{G}_{0} / \mathrm{G}_{1}\right.$ $\left.+\mathrm{S}+\mathrm{G}_{2} / \mathrm{M}\right) \times 100 \%$. 


\section{Assessment of senescence-associated $\beta$-galactosidase staining}

Marrow stromal cells $\mathrm{s}$ were seeded in a 6-well plate with $2 \times 10^{3} \mathrm{cell} / \mathrm{cm}^{2}$. After 2 days of culture, the medium was discarded, cells were rinsed with PBS once, $1 \mathrm{ml}$ of fixative per well was added for $15 \mathrm{~min}$, and subsequently rinsed with PBS 3 times. Then $1 \mathrm{ml}$ per well of working solution of $\beta$-galactosidase with X-Gal was placed and the plate was maintained at $37^{\circ} \mathrm{C}$ overnight (senescence-associated $\beta$-galactosidase staining kit from Beyotime China). The senescent cells were observed in an optical microscope and counted from 5 random fields of vision.

\section{Real-time RT-PCR analysis}

For real-time RT-PCR analysis, cells were harvested and maintained in RNA preservation solution (RNAsafeguard, Keygen, China) before the following experiment. The total RNA was extracted using a simple P total RNA extraction kit (Bioer, China). Total RNA was quantified, in a spectrophotometer, at an absorbance (A) of $260 \mathrm{~nm}$. The RNA samples had an A260 : A280 ratio of 2.0 to guarantee high purity. Two miligrams of total RNA from each sample was subjected to reverse transcription using the SYBR_ PrimeScript ${ }^{T M}$ RT-PCR Kit (TaKaRa, China) according to the manufacturer's protocol. Each real time PCR was carried out in triplicate in a total of $20 \mu$ reaction mixture in an $A B I$ PRISM 7300 Real-time PCR System (Applied Biosystems, US). Primers used for real-time PCR analysis are presented in Table I. The PCR program was conducted according to the suggestion of the Takara manual. The starting copy numbers of unknown samples were calculated by the 7300 System SDS Software (Applied Biosystems) from the standard curve. The housekeeping gene $\beta$-actin was concurrently amplified in each sample as a control and was used for normalization. The cDNA of young adult MSCs with or without osteo-induction normalized to the level of $\beta$-actin was ascribed a fold induction of 1 . Melting curves for each PCR reaction were generated to ensure the purity of the amplification product.

\section{Quantitative alkaline phosphatase and protein assays}

For quantitative analysis of alkaline phosphatase activities, cells after 14 days of osteo-induction were washed with PBS, prepared following the instruction of the alkaline phosphatase (ALP) assay kit (Merit Choice, China). Briefly, cells were lysed with $10 \mathrm{mM}$ Tris- $\mathrm{HCl}$ and $0.1 \%$ Triton $\mathrm{X}-100, \mathrm{pH} 7.4$ and repeating freeze-thaw cycle for 3 times. After sonication, the lysates were taken for assay ALP in a microtitre plate using $p$-nitrophenyl phosphate as the substrate. The absorbance of $p$-nitrophenol formed by the hydrolysis of $p$-nitrophenyl phosphate, catalysed by ALP, was measured at $405 \mathrm{~nm}$ by a microplate reader (HTS 7000 Plus, PerkinElmer, US). Total protein content was measured by the Bradford method, read at $595 \mathrm{~nm}$ and calculated according to the bovine $\gamma$ globulin standards. Alkaline phosphatase activity was expressed as unit/g protein.

\section{Enzyme-linked immunosorbent assay for osteocalcin}

To quantify and compare the concentration of osteocalcin (OCN) in cell cultured supernatant of the three groups of MSCs, commercially available enzyme linked immunosorbent assay (ELISA) kits were used according to the manufacturer's recommendations (R\&D, US). Constitutive OCN secretion was analysed after 2 weeks of osteogenic induction. Prior to collection, the supernatant of the medium was centrifuged to remove cell debris.

\section{Western blotting assay for BMAL1 protein level}

To obtain whole-cell protein extracts, cells were washed twice with ice-cold PBS and then lysed in a lysis buffer (Keygen total protein extraction kit, Keygen Biotech, China). The supernatant was collected after centrifugation at $14,000 \mathrm{~g}$ at $4^{\circ} \mathrm{C}$ for $15 \mathrm{~min}$ and assayed quantitatively with the BCA method. The total protein extracts were subjected to the standard procedure of SDS-PAGE and subsequent transfer to a PVDF membrane. After blocking, the membranes were probed with dilution of the anti-BMAL and anti- $\beta$-actin primary antibody

Table I. qRT-PCR primers

\begin{tabular}{|llcc|}
\hline Target gene & Primers & Sequence & Fragment size [bp] \\
\hline Bmal1 & Forward primer & 5'-AACCTTCCCGCAGCTAACAG-3' & 79 \\
\cline { 2 - 3 } & Reverse primer & 5'-AGTCCTCTTTGGGCCACCTT-3 $^{\prime}$ & \\
\hline Osx & Forward primer & 5'-TATGCTCCGACCTCCTCAAC-3' $^{\prime}$ & 120 \\
\cline { 2 - 3 } & Reverse primer & 5'-AATAGGATTGGGAAGCAGAAA-3' $^{\prime}$ & \\
\hline -actin & Forward primer & 5'-GGGCTGTATTCCCCTCCATCG-3' $^{\prime}$ & 201 \\
\cline { 2 - 3 } & Reverse primer & 5'-GCAGCTCATTGTAGAAGGTGTGGTG-3' & \\
\hline
\end{tabular}


(Abcam), followed by the addition of horseradish peroxidase (HRP)-conjugated secondary antibody. Immunoreactive proteins were visualized using a chemiluminescence kit (Millipore). Band intensities were determined using the ChemiDoc XRS Gel documentation system and Quantity One software (Bio-Rad).

\section{Statistical analysis}

All experiments were conducted a minimum of 3 times. Measurements are expressed as mean \pm SD. Statistical comparisons were made using factorial analysis of variance (ANOVA), followed by multiple comparisons using the SNK test. A value of $p<0.05$ was considered statistically significant.

\section{Results}

\section{Cell culture and osteoblast and adipogenic differentiation}

Marrow stromal cells primary culture and passage 3 culture are shown in Figures 1 A-B. As can be seen, the shape of the cells was more spindlelike after passaging, but we cannot observe any morphological difference among the three groups of MSCs. Besides identification of CD (data not shown), the MSCs isolated from three different ages of animal can all be induced to differentiate to osteoblasts or adipocytes at the stated time by the differentiation medium. The common view under phase contrast microscope after adipo-induction and the result of Alizarin Red, von Kossa and oil red $O$ staining of a sample of MSCs are presented in Figures 2 A-D.

\section{Effects of age on cell proliferation marker and cellular senescence}

Through flow cytometry for detection of cell cycle phase proportion, the results showed that the S-phase fraction rates in 1, 6 and 16 month old mice MSCs were $45.38 \pm 1.52,37.64 \pm 5.72,23.8 \pm 2.91$ per- cent respectively. The aged mice MSCs contained an S-phase fraction rate which was only about half of that in young adult MSCs. The result is presented in Figure $3 \mathrm{~A}$.

Marrow stromal cells isolated from young adult, mid-age and aged mice have a growing rate of SA $\beta$-gal+cells. But both young adult and mid-age mice MSCs presented an SA- $\beta$-gal+ rate lower than $5 \%$, which can be treated as not significant as other researchers suggested [9]. In aged mice, the SA- $\beta$-gal+ cells were more than $10 \%$ of the total count, and showed a slower proliferation rate when passaging (data not shown), which is a sign of susceptibility to toxin in serum or capacity for senescence (see Figure $3 \mathrm{~B}$ ).

\section{Bmal1 and Osx expression affected by age}

Bmal1 gene and protein level dropped along with age, but was much lower in aged mice MSCs. The aged mice MSCs Bmal1 gene and protein level was less than half of the young adult mice MSCs. And the aged mice MSCs Bmal1/ $\beta$-actin protein rate was even less than 1 (Figures $4 \mathrm{~A}-\mathrm{C}$ ).

Osx is a transcription factor that is more strongly expressed in the progenitors of osteoblasts than chondrocytes and also can be detected in MSCs, the progenitors of osteoblasts, chondrocytes and adipocytes. The result of RT-qPCR showed that when MSCs were differentiated into osteoblasts by dexamethasone, $\beta$-glycerophosphate, and L-ascorbate lasting for 2 weeks, their Osx gene level was higher in the young adult and mid-age mice group than the aged mice group (Figure $5 \mathrm{~A}$ ).

\section{Alkaline phosphatase activity and osteocalcin concentration after 2 weeks of osteogenic induction}

Alkaline phosphatase activity level is an early osteoblast marker. When comparing the three groups of cells and detecting the ALP activity level, the results were $15.05 \pm 1.81 \mathrm{U} / \mathrm{g}, 8 \pm 1.44 \mathrm{U} / \mathrm{g}$,
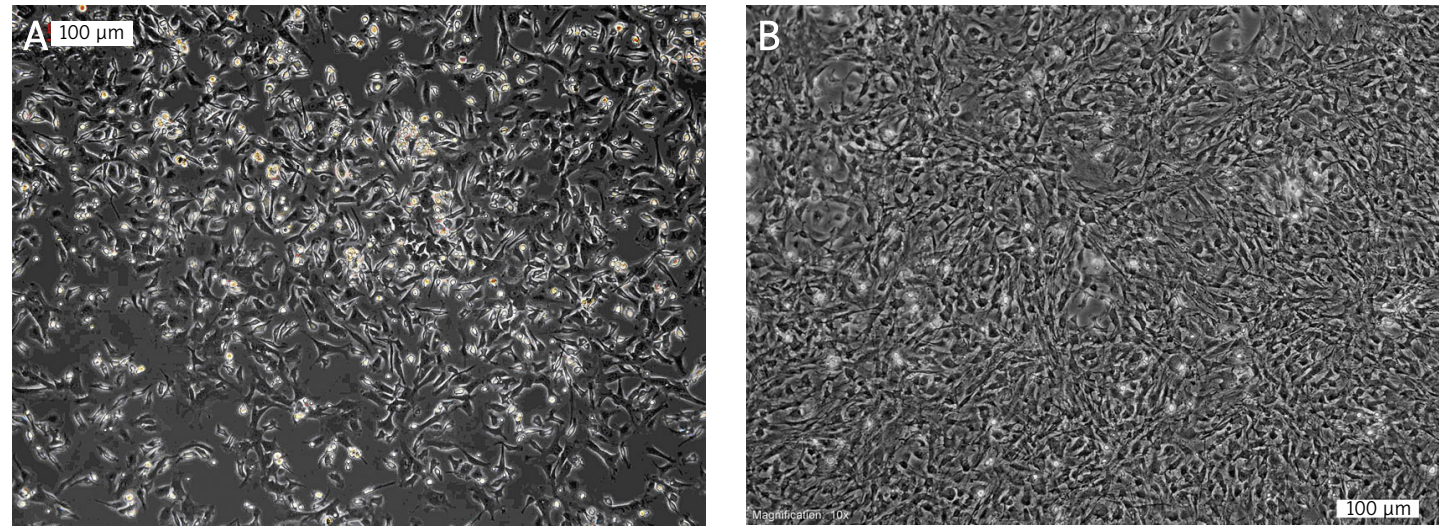

Figure 1. C57BL/6 mouse bone marrow stromal cells primary culture (A) and passage 3 culture (B) maintained in basic culture medium were observed by phase-contrast microscope. Magnification is $100 \times$ 

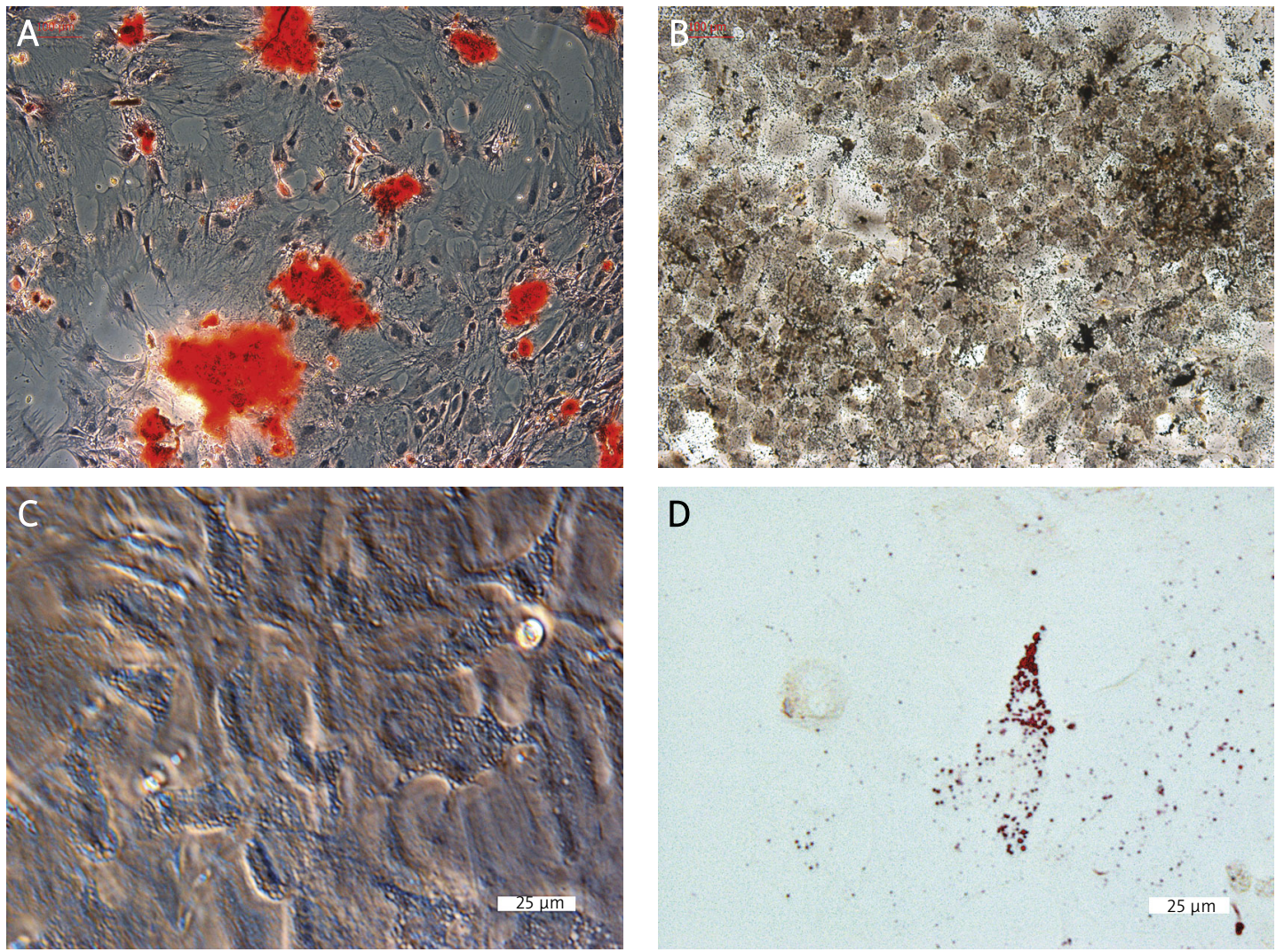

Figure 2. Characterization of C57BL/6 mouse MSCs. Passage 3 MSCs were induced to differentiate to osteoblasts by osteo-induction medium for 3 weeks, stained with Alizarin Red (A), and von Kossa (B). Passage 3 MSCs were induced to adipogenesis by adipo-differentiation medium for 13 days and observed in phase-contrast microscope (C) and stained with oil red O (D). Magnification is $100 \times$ for A, B and $400 \times$ for C, D respectively

A

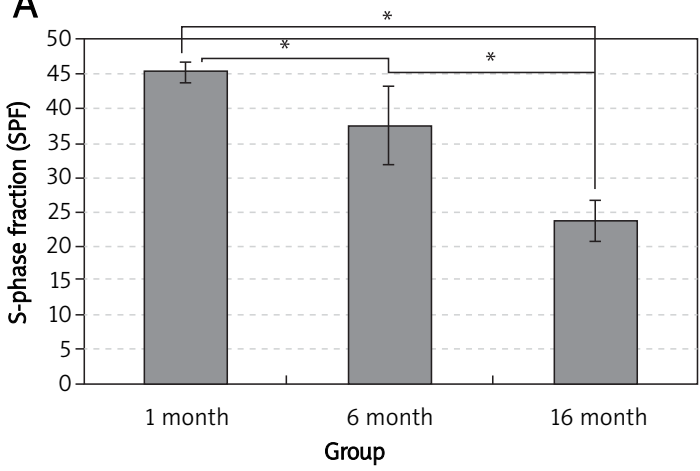

B

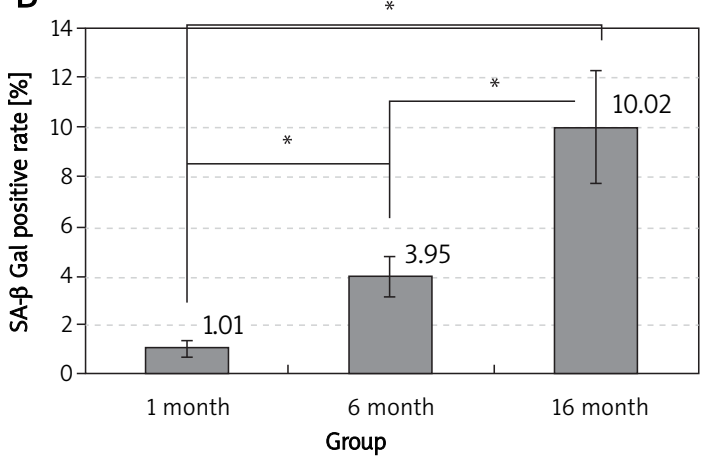

Figure 3. MSCs were investigated on proliferation by flow cytometry and cell senescence by SA- $\beta$-gal. A - Flow cytometry assay indicated that S-phase fraction cell ratio was significantly higher in 1-month group than 6-month group and 6-month group than 16-month group. B - SA- $\beta$-gal assay is a cell senescence marker. The assay indicated that 1 -month and 6-month group MSCs expressed a much lower SA- $\beta$-gal positive cell rate than 16-month group. The values are expressed as means \pm SD of at least 3 independent experiments or 5 random fields of vision ${ }^{*} p<0.05$ means there is a significant difference between them

$3.35 \pm 1.43 \mathrm{U} / g$ protein. The mid-age and aged groups were much less than the young adult group.

Osteocalcin level in the supernatant of culture medium is a late osteogenic marker for detection of mineralization ability. Osteocalcin contents were $58.24 \pm 3.06,47.49 \pm 3.54$ and $18.65 \pm 3.53 \mathrm{ng} / \mathrm{ml}$ from young adult to aged mice MSCs. The OCN content in aged mice group was less than $1 / 3$ of that in the young adult group. Details are provided in Figures $5 \mathrm{~B}$ and $\mathrm{C}$.

\section{Discussion}

The strategy of this experiment was not only to compare young adult and aged mice MSCs' proliferation and osteo-differentiation potential, but also 
A

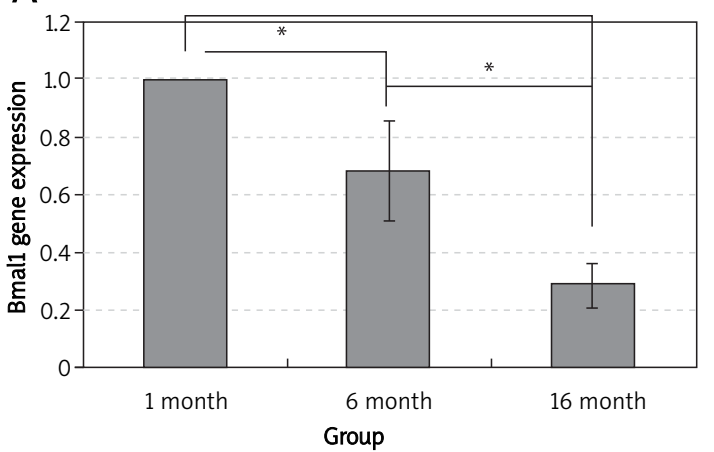

B
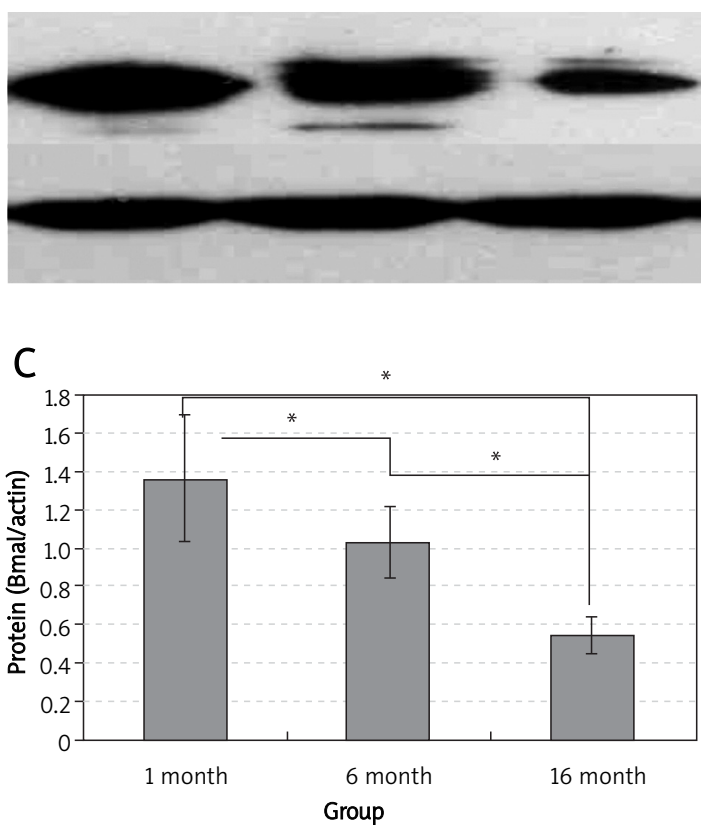

Figure 4. Bmal1 gene and protein level in the three groups of C57BL/ 6 mouse MSCs. A - qRT-PCR results indicate there was much lower Bmal1 mRNA expression in 6-month and 16-month groups of MSCs than 1-month group. The 16-month group's Bmal1 mRNA level was less than $1 / 3$ of that in 1 month group. The cDNA of 1-month group MSCs normalized to the level of $\beta$-actin was ascribed as a fold induction of 1 . B, C - Western blotting results showed the same trend of Bmal1 expression in the three groups. The protein levels were calculated by Bmal $1 / \beta$-actin protein expression ratio. The values are expressed as means \pm SD of at least 3 independent experiments ${ }^{*} p<0.05$ means there is a significant difference between them

to add a mid-age group, because we believe the aging procedure advances gradually. As expected, the results of this study revealed that along with aging, MSCS of C57BL/6 mice expressed more SA- $\beta$-gal, especially in aged mice; with the reverse trend, the $S$-phase fraction ratio of all cell cycles and the Bmal1 gene and protein level dropped. When cells were induced to differentiate to osteoblasts, the three osteo-differentiation markers Osx, ALP
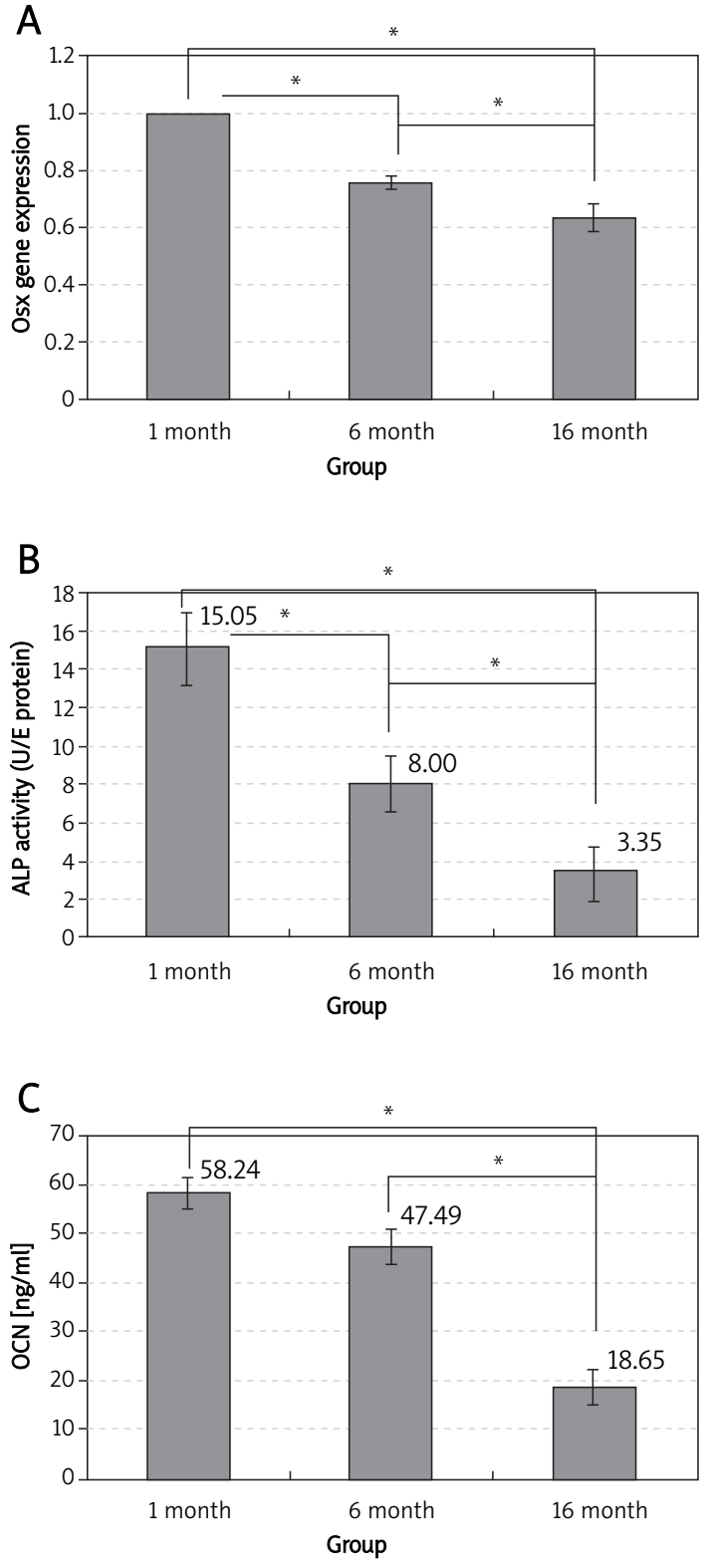

Figure 5. Osteoblast marker Osx, ALP and OCN expression on cultured cells or supernatant of culture medium after 2 weeks of maintenance in osteoinduction medium. A - Osx is an osteogenesis related transcription factor. The MSCs Osx mRNA level showed a decreasing trend, 6-month to 1-month group and 16-month to 6-month group. The CDNA of 1-month group MSCs normalized to the level of $\beta$-actin was ascribed as a fold induction of 1. B - ALP is an early marker of osteogenesis. The ALP activity in MSCs cell lysates indicated that the level in 6month group and 16-month group were about half and below $1 / 4$ of that in 1-month group respectively. C - The OCN is a late osteoblast maturation marker. The OCN protein level in the supernatant of culture medium was calculated by ELISA. The OCN concentration was about $18.65 \mathrm{ng} / \mathrm{ml}$ in 16 -month group MSCs compared to $47.49 \mathrm{ng} / \mathrm{ml}$ in 6-month group and $58.24 \mathrm{ng} / \mathrm{ml}$ in 1-month group. The values are expressed as means \pm SD of at least 3 independent experiments

${ }^{*} p<0.05$ means there is a significant difference between them 
and OCN presented a consistent trend, but the proportion of each age group was not exactly the same. The Osx level decreased along with the aging process, ALP activity was much higher in MSCs of young adult mice, and OCN protein synthesis was dramatically lower in MSCs of aged mice.

To find out whether there are intrinsic, senescence-associated changes of MSCs in vivo, we evaluated the freshly isolated stromal cells at very early passage. This strategy was intended to avoid changes in cell behaviours that are related to prolonged culture [10]. Like many other types of mammalian cells, MSCs with repeated passaging display "in vitro senescence", such as decreased proliferation, replicative quiescence, enlargement and increased SA- $\beta$-gal activity [11]. This in vitro method was also intended to eliminate the aged-related extrinsic somatic environment change, i.e. declines in circulating hormones [12] when applying in vivo study, and to focus on the intrinsic aging changes themselves.

In the present study, there was a much lower frequency of SA- $\beta$-gal positive cells and much higher $S$-phase fraction of the cell cycle in young adult and mid-age mice MSCs, compared with the aged one. In fact, the frequency of SA- $\beta$-gal positive cells in the former two age groups can be ignored because the frequency was less than $5 \%$. In older mice, the early passage MSCs showed a frequency of $10 \%$ of SA- $\beta$-gal positive cells, suggesting an age threshold of abundantly showing this marker, suggesting that when aging, some MSCs fail to protect themselves from metabolic waste or an increased level of reactive oxygen species, etc [13]. The S-phase fraction is a measure of the percentage of cells that are in the phase of DNA synthesis and represents a proliferative index of cell culture. The result of SPF detection was consistent with our observation that aged mice MSCs needed more time to reach confluence than the other two groups under the same culture conditions.

We detected the Bmal1 mRNA and protein level in MSCs of the three groups. Its trend was as the SPF ratio and as the reverse transformation of SA- $\beta$-gal+ ratio. The core circadian protein BMAL1, in addition to its known functions in the circadian oscillator, plays essential non-redundant roles in the control of tissue homeostasis and aging. One of the most striking phenomena was premature aging of Bmal1-/- animals [3]. While there are no morphological differences between Bmal1-/- animals and their wild-type littermates at birth and early age, 18-week-old Bmal1-/- mice showed signs of growth retardation and 36-week-old knockout mice morphologically resembled aged mice. The study suggested that the control of aging is a novel function of Bmal1, relatively independent from its role in the circadian oscillator. Along with our study results, the Bmal1 gene and protein level were much lower in older mice's MSCs, and the frequency of SA- $\beta$-gal positive cells was much higher as well. We can speculate that Bmal1 reduction or malfunction can also be a candidate reason of MSCs in vivo ageing or cell senescence [14]. Because BMAL1 was reported to participate in controlling glucose and fat metabolism and homeostasis [15, $16]$, and MSCs are the progenitors of adipocytes, chondrocytes and osteoblasts, the hypothesis may be convincing. Recently, studies showed that cyclindependent kinase inhibitor p21, which negatively regulates the G1/S phase transition, is antagonized by Bmal1 through the orphan nuclear receptor ROR/REV-ERB pathway with or without a relationship with p21's regulator, p53 [17, 18]. These investigations established a novel molecular link between clock and cell cycle genes. Because ROR and REV-ERB are BMAL1's activator and repressor respectively, the three transcriptional factors can form an interlock loop that plays a stabilizing role in circadian rhythm $[19,20]$. This may be the reason why the aged mice MSCs showed a much more decreased Bmal1 level and SPF rate as well.

In aging, bone formation activity is significantly reduced. It has been postulated that a deficiency in osteoprogenitor cells is the main reason for the decline in bone induction activity [21]. This is because osteoprogenitor cells play a major role not only in bone formation but also in bone resorption $[22,23]$. In contrast, a few researchers found no effects of age-related decline in osteoblast potential when applying colony assays for the numbers of colonies that stain for alkaline phosphatase [24]. Osteoblast differentiation is regulated by a complex network of transcription factors and different signalling pathways. Osteoblastic differentiation in vitro is also characterized by three distinct stages of cellular activity: proliferation, extracellular matrix (ECM) maturation and mineralization $[25,26]$. So we recruited three indices of osteoblast differentiation: transcriptional factor Osx, early osteogenic marker ALP and late osteogenic marker OCN, hypothesizing that their aging evolvement would be different. As we expected, the Osx level in MSCs decreased gradually with aging of the mice, revealing a different response to osteogenic activation. The early osteoblast marker ALP activity was also higher in young adult mice MSCs, but much lower in mid-age and aged mice MSCs. However, while ALP level was much lower in mid-age MSCs than young adult MSCs, the late osteoblast marker, OCN level, was not statistically different between the two groups, and it needs to be stressed that the aged mice showed the dramatically lowest level of OCN. Our research results showed a gradual change of osteogenic ability along with aging with osteodifferentiation indices. 
While some investigators believe that the reduction of bone mass in older persons is not caused by a shift in differentiation of these precursors towards adipocytes, others did not agree with that viewpoint $[27,28]$. If so, there must be some aging intrinsic changes or signalling pathway with respect to their differentiation potential. Aging alters the circadian biology in several aspects including the circadian components [29] and there have been clues linking circadian rhythms to bone metabolism: clinical studies have detected 24-h oscillations in human serum levels of osteocalcin and alkaline phosphatase [30, 31]. A recent study showed that a circadian gene could mediate leptin-dependent bone formation [32]. CLOCK and BMAL1 proteins heterodimerize to form a transcriptional factor but the CLOCK mutant did not show the same symptom of pre-aging as Bmal1-/- mice; this indicated that Bmal1 decided the complete functionality of the complex or else BMAL1 deficiency would affect activities of different systems [33, 34]. The circadian clock affects physiological processes through the regulation of the clock-controlled gene or output gene. The clock-controlled genes are tissue-specific and some osteogenic related genes can be controlled by the circadian clock or Bmal1 in bone marrow and MSCs [5, 35]. In another way, the mechanism of Bmal1 controlling aged osteogenic potential change is still unclear. Recent information showed that ROR, a positive regulator and target of Bmal1, must be an imperative modulator of bone sialoprotein and dentin matrix protein 1 involved in the osteoblast differentiation and bone formation. And Bmal1's other antagonized target, p53, is a negative regulator of osteoblast differentiation [36-38]. These can be clues for future study.

We have shown the normal aging effects on murine MSCs proliferation and differentiation, and with the aforementioned information in mind, we concluded that besides its effects on mice MSCs proliferation, Bmal1 could play a role in the late stage of osteoblast maturation and mineralization. The results must be further tested using murine models harbouring conditional alleles of Bmal1 that allow tissue-specific deletion or overexpression as well as in osteoblasts or MSCs.

\section{Acknowledgments}

This work was supported by a grant from the National Natural Science Foundation of China (No. 30801206).

\section{References}

1. Merrot O, Vacher C, Merrot S, Godlewski G, Frigard B, Goudot P. Changes in the edentate mandible in the elderly. Surg Radiol Anat 2005; 27: 265-70.

2. Kashani IR, Zaminy A, Barbarestani $M$, et al. In vitro osteogenesis of rat adipose-derived stem cells: com- parison with bone marrow stem cells. Arch Med Sci 2009; 5: 149-55.

3. Kondratov RV, Kondratova AA, Gorbacheva VY, Vykhovanets OV, Antoch MP. Early aging and age-related pathologies in mice deficient in BMAL1, the core component of the circadian clock. Genes Dev 2006; 20: 1868-73.

4. Langmesser S, Tallone T, Bordon A, Rusconi S, Albrecht U. Interaction of circadian clock proteins PER2 and CRY with BMAL1 and CLOCK. BMC Mol Biol 2008; 9: 41-56.

5. McDearmon EL, Patel KN, Ko CH, et al. Dissecting the functions of the mammalian clock protein BMAL1 by tissue-specific rescue in mice. Science 2006; 314 : 1304-8.

6. Wu X, Yu G, Parks H, et al. Circadian mechanisms in murine and human bone marrow mesenchymal stem cells following dexamethasone exposure. Bone 2008; 42: 861-70.

7. Friedenstein AJ, Latzinik NV, Gorskaya Yu F, Luria EA, Moskvina IL. Bone marrow stromal colony formation requires stimulation by haemopoietic cells. Bone Miner 1992; 18: 199-213.

8. Bianco P, Riminucci M, Gronthos S, Robey PG. Bone marrow stromal stem cells: nature, biology, and potential applications. Stem Cells 2001; 19: 180-92.

9. Zhou S, Greenberger JS, Epperly MW, et al. Age-related intrinsic changes in human bone-marrow-derived mesenchymal stem cells and their differentiation to osteoblasts. Aging Cell 2008; 7: 335-43.

10. Gou S, Wang C, Liu T, et al. Spontaneous differentiation of murine bone marrow-derived mesenchymal stem cells into adipocytes without malignant transformation after long-term culture. Cells Tissues Organs 2010; 191: 185-92.

11. Sethe S, Scutt A, Stolzing A. Aging of mesenchymal stem cells. Ageing Res Rev 2006; 5: 91-116.

12. Edelberg JM, Ballard VL Stem cell review series: regulating highly potent stem cells in aging: environmental influences on plasticity. Aging Cell 2008; 7: 599-604.

13. Balaban RS, Nemoto S, Finkel T. Mitochondria, oxidants, and aging. Cell 2005; 120: 483-95.

14. Kunieda T, Minamino T, Katsuno T, et al. Cellular senescence impairs circadian expression of clock genes in vitro and in vivo. Circ Res 2006; 98: 532-9.

15. Rudic RD, McNamara P, Curtis AM, et al. BMAL1 and CLOCK, two essential components of the circadian clock, are involved in glucose homeostasis. PLoS Biol 2004; 2: e377.

16. Shimba S, Ishii N, Ohta Y, et al. Brain and muscle Arnt-like protein-1 (BMAL1), a component of the molecular clock, regulates adipogenesis. Proc Natl Acad Sci U S A 2005; 102: 12071-6.

17. Grechez-Cassiau A, Rayet B, Guillaumond F, Teboul M, Delaunay F. The circadian clock component BMAL1 is a critical regulator of p21WAF1/CIP1 expression and hepatocyte proliferation. J Biol Chem 2008; 283: 4535-42.

18. Mullenders J, Fabius AW, Madiredjo M, Bernards R, Beijersbergen RL. A large scale shRNA barcode screen identifies the circadian clock component ARNTL as putative regulator of the $\mathrm{p} 53$ tumor suppressor pathway. PLoS One 2009; 4: e4798.

19. Preitner N, Damiola F, Lopez-Molina L, et al. The orphan nuclear receptor REV-ERBalpha controls circadian transcription within the positive limb of the mammalian circadian oscillator. Cell 2002; 110: 251-60.

20. Nakajima Y, Ikeda M, Kimura T, Honma S, Ohmiya Y, Honma K. Bidirectional role of orphan nuclear receptor RORalpha in clock gene transcriptions demonstrated 
by a novel reporter assay system. FEBS Lett 2004; 565: 122-6.

21. Wlodarski KH, Wlodarski PK, Galus R. Senescence of osteogenic cells. Review [Polish]. Ortop Traumatol Rehabil 2007; 9: 63-7.

22. Fili S, Karalaki M, Schaller B. Therapeutic implications of osteoprotegerin. Cancer Cell Int 2009; 9; 26.

23. Fili S, Karalaki M, Schaller B. Mechanism of bone metastasis: the role of osteoprotegerin and of the hosttissue microenvironment-related survival factors. Cancer Lett 2009; 283: 10-9.

24. Dobson K, Reading L, Scutt A. A cost-effective method for the automatic quantitative analysis of fibroblastic colonyforming units. Calcif Tissue Int 1999; 65: 166-72.

25. Lian JB, Stein GS, Javed A, et al. Networks and hubs for the transcriptional control of osteoblastogenesis. Rev Endocr Metab Disord 2006; 7: 1-16.

26. Sila-Asna M, Bunyaratvej A, Maeda S, Kitaguchi H, Bunyaratavej $\mathrm{N}$. Osteoblast differentiation and bone formation gene expression in strontium-inducing bone marrow mesenchymal stem cell. Kobe J Med Sci 2007; 53: 25-35.

27. Moerman EJ, Teng K, Lipschitz DA, Lecka-Czernik B. Aging activates adipogenic and suppresses osteogenic programs in mesenchymal marrow stroma/stem cells: the role of PPAR-gamma2 transcription factor and TGF-beta/BMP signaling pathways. Aging Cell 2004; 3: 379-89.

28. Kretlow JD, Jin YQ, Liu W, et al. Donor age and cell passage affects differentiation potential of murine bone marrowderived stem cells. BMC Cell Biol 2008; 9: 60-72.

29. Kolker DE, Fukuyama H, Huang DS, Takahashi JS, Horton $\mathrm{TH}$, Turek FW. Aging alters circadian and light-induced expression of clock genes in golden hamsters. J Biol Rhythms 2003; 18: 159-69.

30. Gundberg CM, Markowitz ME, Mizruchi M, Rosen JF. Osteocalcin in human serum: a circadian rhythm. J Clin Endocrinol Metab 1985; 60: 736-9.

31. Shao P, Ohtsuka-Isoya M, Shinoda H. Circadian rhythms in serum bone markers and their relation to the effect of etidronate in rats. Chronobiol Int 2003; 20: 325-36.

32. Fu L, Patel MS, Bradley A, Wagner EF, Karsenty G. The molecular clock mediates leptin-regulated bone formation. Cell 2005; 122: 803-15.

33. Kwon I, Lee J, Chang SH, et al. BMAL1 shuttling controls transactivation and degradation of the CLOCK/BMAL1 heterodimer. Mol Cell Biol 2006; 26: 7318-30.

34. Kondratov RV. A role of the circadian system and circadian proteins in aging. Ageing Res Rev 2007; 6: 12-27.

35. Liu AC, Tran HG, Zhang EE, Priest AA, Welsh DK, Kay SA. Redundant function of REV-ERBalpha and beta and nonessential role for Bmal1 cycling in transcriptional regulation of intracellular circadian rhythms. PLoS Genet 2008; 4: e1000023.

36. Miyamoto S, Cooper L, Watanabe K, et al. Role of retinoic acid-related orphan receptor-alpha in differentiation of human mesenchymal stem cells along with osteoblastic lineage. Pathobiology 2010; 77: 28-37.

37. Wang X, Kua HY, Hu Y, et al. p53 functions as a negative regulator of osteoblastogenesis, osteoblast-dependent osteoclastogenesis, and bone remodeling. J Cell Biol 2006; 172: 115-25.

38. Lengner CJ, Steinman HA, Gagnon J, et al. Osteoblast differentiation and skeletal development are regulated by Mdm2-p53 signaling. J Cell Biol 2006; 172: 909-21. 